Pacific

Journal of

Mathematics

UNIQUENESS OF THE CHEEGER SET OF A CONVEX BODY

Vicent Caselles, Antonin Chambolle and Matteo Novaga 


\title{
UNIQUENESS OF THE CHEEGER SET OF A CONVEX BODY
}

\author{
Vicent Caselles, Antonin Chambolle and Matteo Novaga
}

\begin{abstract}
We prove that if $C \subset \mathbb{R}^{N}$ is of class $C^{2}$ and uniformly convex, the Cheeger set of $C$ is unique. The Cheeger set of $C$ is the set that minimizes, inside $C$, the ratio of perimeter over volume.
\end{abstract}

\section{Introduction}

For a nonempty open bounded subset $\Omega$ of $\mathbb{R}^{N}$, the Cheeger constant of $\Omega$ is the quantity

$$
h_{\Omega}=\min _{K \subseteq \Omega} \frac{P(K)}{|K|} .
$$

Here $|K|$ denotes the $N$-dimensional volume of $K$ and $P(K)$ denotes the perimeter of $K$. The minimum in (1) is taken over all nonempty sets of finite perimeter contained in $\Omega$. A Cheeger set of $\Omega$ is any set $G \subseteq \Omega$ which minimizes (1). If $\Omega$ minimizes (1), we say that it is Cheeger in itself. We observe that the minimum in (1) is attained at a subset $G$ of $\Omega$ such that $\partial G$ intersects $\partial \Omega$ : otherwise we could diminish the quotient $P(G) /|G|$ by dilating $G$.

For any set $K$ of finite perimeter in $\mathbb{R}^{N}$, define

$$
\lambda_{K}:=\frac{P(K)}{|K|} .
$$

Thus $\lambda_{G}=h_{G}$ for any Cheeger set $G$ of $\Omega$. Moreover, $G$ is a Cheeger set of $\Omega$ if and only if $G$ minimizes

$$
\min _{K \subseteq \Omega} P(K)-\lambda_{G}|K|
$$

We say that a set $\Omega \subset \mathbb{R}^{N}$ is calibrable if $\Omega$ minimizes the problem

$$
\min _{K \subseteq \Omega} P(K)-\lambda_{\Omega}|K| \text {. }
$$

MSC2000: primary 35J70; secondary 49J40, 52A20.

Keywords: convex bodies, variational problems.

Caselles was partially support by the Departament d'Universitats, Recerca i Societat de la Informació de la Generalitat de Catalunya and by PNPGC project, reference BFM2003-02125. 
Any Cheeger set $G$ of $\Omega$ is clearly calibrable. Thus, $\Omega$ is a Cheeger set of itself if and only if it is calibrable.

Finding the Cheeger sets of a given $\Omega$ is a difficult task. The task is simplified if $\Omega$ is a convex set and $N=2$. In that case, the Cheeger set of $\Omega$ is unique and equals the set $\Omega^{R} \oplus B(0, R)$, where $\Omega^{R}:=\{x \in \Omega: \operatorname{dist}(x, \partial \Omega)>R\}$ is such that $\left|\Omega^{R}\right|=\pi R^{2}$ and $A \oplus B:=\{a+b: a \in A, b \in B\}$, for $A, B \subset \mathbb{R}^{2}$ [Alter et al. 2005b; Kawohl and Lachand-Robert 2006]. In particular, in this case the Cheeger set is convex.

A convex set $\Omega \subseteq \mathbb{R}^{2}$ is Cheeger in itself if and only if ess $\sup _{x \in \partial \Omega} \kappa_{\Omega}(x) \leq \lambda_{\Omega}$, where $\kappa_{\Omega}(x)$ denotes the curvature of $\partial \Omega$ at the point $x$. This has been proved in [Giusti 1978; Bellettini et al. 2002; Kawohl and Lachand-Robert 2006; Alter et al. 2005b; Kawohl and Novaga 2006], though it was stated in terms of calibrability in the second and fourth of these references. The proof in [Giusti 1978] had a complementary result: if $\Omega$ is Cheeger in itself then $\Omega$ is strictly calibrable, that is, for any set $K \subsetneq \Omega$, we have

$$
0=P(\Omega)-\lambda_{\Omega}|\Omega|<P(K)-\lambda_{\Omega}|K| .
$$

(This implies that the gravity-less capillary problem with vertical contact angle at the boundary, given by

$$
\begin{aligned}
-\operatorname{div} \frac{D u}{\sqrt{1+|D u|^{2}}}=\lambda \Omega & \text { in } \Omega, \\
-\frac{D u}{\sqrt{1+|D u|^{2}}} \cdot v^{\Omega}=1 & \text { in } \partial \Omega,
\end{aligned}
$$

has a solution. Indeed, the two problems are equivalent [Giusti 1978; Kawohl and Kutev 1995].)

Our purpose in this paper is to extend the preceding result to $\mathbb{R}^{N}$, that is, to prove the uniqueness and convexity of the Cheeger set contained in a convex set $\Omega \subset \mathbb{R}^{N}$. We have to assume, in addition, that $\Omega$ is uniformly convex and of class $C^{2}$. This regularity assumption is probably too strong, and its removal is the subject of current research [Alter and Caselles 2007]. The characterization of a convex set $\Omega \subset \mathbb{R}^{N}$ of class $C^{1,1}$ which is Cheeger in itself (also called calibrable) in terms of the mean curvature of its boundary was proved in [Alter et al. 2005a]. The precise result states that such a set $\Omega$ is Cheeger in itself if and only if $\kappa_{\Omega}(x) \leq \lambda_{\Omega}$ for almost any $x \in \partial \Omega$, where $\kappa_{\Omega}(x)$ denotes the sum of the principal curvatures of the boundary of $\Omega$, which is to say, $N-1$ times the mean curvature of $\partial \Omega$ at $x$. In [Alter et al. 2005a] it was also proved that for any convex set $\Omega \subset \mathbb{R}^{N}$ there exists a maximal Cheeger set contained in $\Omega$ which is convex. These results were extended to convex sets $\Omega$ satisfying a regularity condition and anisotropic norms in $\mathbb{R}^{N}$ (including the crystalline case) in [Caselles et al. 2005]. 
In particular, we obtain that $\Omega \subset \mathbb{R}^{N}$ is the unique Cheeger set of itself, whenever $\Omega$ is a $C^{2}$, uniformly convex calibrable set. We point out that, by Theorems 1.1 and 4.2 in [Giusti 1978], this uniqueness result is equivalent to the existence of a solution $u \in W_{\text {loc }}^{1, \infty}(\Omega)$ of the capillary problem (4).

In Section 2 we collect some definitions and recall results about the mean curvature operator in (4) and the subdifferential of the total variation. In Section 3 we state and prove our uniqueness result.

\section{Preliminaries}

2.1. BV functions. Let $\Omega$ be an open subset of $\mathbb{R}^{N}$. A function $u \in L^{1}(\Omega)$ whose gradient $D u$ in the sense of distributions is a (vector valued) Radon measure with finite total variation in $\Omega$ is called a function of bounded variation. The class of such functions will be denoted by $B V(\Omega)$. The total variation of $D u$ on $\Omega$ turns out to be

(5) $\sup \left\{\int_{\Omega} u \operatorname{div} z d x: z \in C_{0}^{\infty}\left(\Omega ; \mathbb{R}^{N}\right),\|z\|_{L^{\infty}(\Omega)}:=\operatorname{ess}_{\sup _{x \in \Omega}}|z(x)| \leq 1\right\}$,

(where for a vector $v=\left(v_{1}, \ldots, v_{N}\right) \in \mathbb{R}^{N}$ we set $|v|^{2}:=\sum_{i=1}^{N} v_{i}^{2}$ ) and will be denoted by $|D u|(\Omega)$ or by $\int_{\Omega}|D u|$. The map $u \mapsto|D u|(\Omega)$ is $L_{\text {loc }}^{1}(\Omega)$-lower semicontinuous. $B V(\Omega)$ is a Banach space when endowed with the norm $\int_{\Omega}|u| d x+$ $|D u|(\Omega)$. We recall that $B V\left(\mathbb{R}^{N}\right) \subseteq L^{N /(N-1)}\left(\mathbb{R}^{N}\right)$.

A measurable set $E \subseteq \mathbb{R}^{N}$ is said to be of finite perimeter in $\mathbb{R}^{N}$ if (5) is finite when we substitute for $u$ the characteristic function $\chi_{E}$ of $E$ and $\Omega=\mathbb{R}^{N}$. The perimeter of $E$ is defined as $P(E):=\left|D \chi_{E}\right|\left(\mathbb{R}^{N}\right)$. For more information on functions of bounded variation we refer to [Ambrosio et al. 2000].

Finally, we denote by $\mathscr{H}^{N-1}$ the $(N-1)$-dimensional Hausdorff measure. We recall that when $E$ is a finite-perimeter set with regular boundary (for instance, Lipschitz), its perimeter $P(E)$ also coincides with the more standard definition $\mathscr{H}^{N-1}(\partial E)$.

2.2. A generalized Green's formula. Let $\Omega$ be an open subset of $\mathbb{R}^{N}$. Following [Anzellotti 1983a], let

$$
X_{2}(\Omega):=\left\{z \in L^{\infty}\left(\Omega ; \mathbb{R}^{N}\right): \operatorname{div} z \in L^{2}(\Omega)\right\} .
$$

If $z \in X_{2}(\Omega)$ and $w \in L^{2}(\Omega) \cap B V(\Omega)$ we define the functional

$$
(z \cdot D w): C_{0}^{\infty}(\Omega) \rightarrow \mathbb{R}
$$

by the formula

$$
\langle(z \cdot D w), \varphi\rangle:=-\int_{\Omega} w \varphi \operatorname{div} z d x-\int_{\Omega} w z \cdot \nabla \varphi d x .
$$


Then $(z \cdot D w)$ is a Radon measure in $\Omega$,

$$
\int_{\Omega}(z \cdot D w)=\int_{\Omega} z \cdot \nabla w d x \quad \text { for } w \in L^{2}(\Omega) \cap W^{1,1}(\Omega) .
$$

Recall that the outer unit normal to a point $x \in \partial \Omega$ is denoted by $v^{\Omega}(x)$. We recall the following result proved in [Anzellotti 1983a].

Theorem 1. Let $\Omega \subset \mathbb{R}^{N}$ be a bounded open set with Lipschitz boundary. Let $z \in$ $X_{2}(\Omega)$. Then there exists a function $\left[z \cdot v^{\Omega}\right] \in L^{\infty}(\partial \Omega)$ satisfying $\left\|\left[z \cdot v^{\Omega}\right]\right\|_{L^{\infty}(\partial \Omega)} \leq$ $\|z\|_{L^{\infty}\left(\Omega ; \mathbb{R}^{N}\right)}$, and such that for any $u \in B V(\Omega) \cap L^{2}(\Omega)$ we have

$$
\int_{\Omega} u \operatorname{div} z d x+\int_{\Omega}(z \cdot D u)=\int_{\partial \Omega}\left[z \cdot v^{\Omega}\right] u d \mathscr{H}^{N-1} .
$$

Moreover, if $\varphi \in C^{1}(\bar{\Omega})$ then $\left[(\varphi z) \cdot v^{\Omega}\right]=\varphi\left[z \cdot v^{\Omega}\right]$.

This result is complemented with the following.

Theorem 2 [Anzellotti 1983b]. Let $\Omega \subset \mathbb{R}^{N}$ be a bounded open set with a boundary of class $C^{1}$. Let $z \in C\left(\bar{\Omega} ; \mathbb{R}^{N}\right)$ with $\operatorname{div} z \in L^{2}(\Omega)$. Then

$$
\left[z \cdot v^{\Omega}\right](x)=z(x) \cdot v^{\Omega}(x) \quad \mathscr{H}^{N-1} \text {-a.e. on } \partial \Omega .
$$

2.3. Some auxiliary results. Let $\Omega$ be an open bounded subset of $\mathbb{R}^{N}$ with Lipschitz boundary, and let $\varphi \in L^{1}(\Omega)$. For all $\epsilon>0$, we let $\Psi_{\varphi}^{\varepsilon}: L^{2}(\Omega) \rightarrow(-\infty,+\infty]$ be the functional defined by

$$
\Psi_{\varphi}^{\epsilon}(u):=\left\{\begin{array}{cl}
\int_{\Omega} \sqrt{\epsilon^{2}+|D u|^{2}}+\int_{\partial \Omega}|u-\varphi| & \text { if } u \in L^{2}(\Omega) \cap B V(\Omega), \\
+\infty & \text { if } u \in L^{2}(\Omega) \backslash B V(\Omega) .
\end{array}\right.
$$

As it is proved in [Giusti 1976], if $f \in W^{1, \infty}(\Omega)$, then the minimum $u \in B V(\Omega)$ of the functional

$$
\Psi_{\varphi}^{\epsilon}(u)+\int_{\Omega}|u(x)-f(x)|^{2} d x
$$

belongs to $u \in C^{2+\alpha}(\Omega)$, for every $\alpha<1$. The minimum $u$ of (7) is a solution of

$$
\begin{cases}u-\frac{1}{\lambda} \operatorname{div} \frac{D u}{\sqrt{\varepsilon^{2}+|D u|^{2}}}=f(x) & \text { in } \Omega, \\ u=\varphi & \text { on } \partial \Omega\end{cases}
$$

where the boundary condition is taken in a generalized sense [Lichnewsky and Temam 1978], i.e.,

$$
\left[\frac{D u}{\sqrt{\varepsilon^{2}+|D u|^{2}}} \cdot v^{\Omega}\right] \in \operatorname{sign}(\varphi-u) \quad \mathscr{H}^{N-1} \text {-a.e. on } \partial \Omega .
$$


Observe that (8) can be written as

$$
u+\frac{1}{\lambda} \partial \Psi_{\varphi}^{\epsilon}(u) \ni f .
$$

We are particularly interested in the case where $\varphi=0$. As we shall show below (see also [Alter et al. 2005a]) in the case of interest to us we have $u>0$ on $\partial \Omega$ and thus,

$$
\left[\frac{D u}{\sqrt{\varepsilon^{2}+|D u|^{2}}} \cdot v^{\Omega}\right]=-1 \quad \mathscr{H}^{N-1} \text {-a.e. on } \partial \Omega .
$$

It follows that $u$ is a solution of the first equation in (8) with vertical contact angle at the boundary.

As $\epsilon \rightarrow 0^{+}$, the solution of (8) converges to the solution of

$$
\begin{cases}u+\frac{1}{\lambda} \partial \Psi_{\varphi}(u)=f(x) & \text { in } \Omega \\ u=\varphi & \text { on } \partial \Omega .\end{cases}
$$

where $\Psi: L^{2}(\Omega) \rightarrow(-\infty,+\infty]$ is given by

$$
\Psi_{\varphi}(u):=\left\{\begin{array}{cl}
\int_{\mathbb{R}^{N}}|D u|+\int_{\partial \Omega}|u-\varphi| & \text { if } u \in L^{2}(\Omega) \cap B V(\Omega), \\
+\infty & \text { if } u \in L^{2}(\Omega) \backslash B V(\Omega) .
\end{array}\right.
$$

In this case $\partial \Psi_{\varphi}$ represents the operator $-\operatorname{div} \frac{D u}{|D u|}$ with the boundary condition $u=\varphi$ in $\partial \Omega$, as shown by:

Lemma 2.1 [Andreu et al. 2001]. The following assertions are equivalent:

(a) $v \in \partial \Psi_{\varphi}(u)$.

(b) $u \in L^{2}(\Omega) \cap B V(\Omega), v \in L^{2}(\Omega)$, and there exists $z \in X_{2}(\Omega)$ with $\|z\|_{\infty} \leq 1$, such that $v=-\operatorname{div} z$ in $\mathscr{D}^{\prime}(\Omega), z \cdot D u=|D u|$, and

$$
\left[z \cdot v^{\Omega}\right] \in \operatorname{sign}(\varphi-u) \quad \mathscr{H}^{N-1} \text {-a.e. on } \partial \Omega .
$$

Notice that the solution $u \in L^{2}(\Omega)$ of (10) minimizes the problem

(12) $\min _{u \in B V(\Omega)} \int_{\Omega}|D u|+\int_{\partial \Omega}|u(x)-\varphi(x)| d \mathscr{H}^{N-1}(x)+\frac{\lambda}{2} \int_{\Omega}|u(x)-f(x)|^{2} d x$, and the two problems are equivalent.

\section{The uniqueness theorem}

We now state our main result.

Theorem 3. Let $C$ be a convex body in $\mathbb{R}^{N}$. Assume that $C$ is uniformly convex, with boundary of class $C^{2}$. Then the Cheeger set of $C$ is convex and unique. 
We do not believe that the regularity and the uniform convexity of $C$ is essential for this result (see [Alter and Caselles 2007]).

Theorem 4 [Alter et al. 2005a, Theorems 6 and 8, Proposition 4]. Let $C$ be a convex body in $\mathbb{R}^{N}$ with boundary of class $C^{1,1}$. For any $\lambda, \varepsilon>0$, there is a unique solution $u_{\varepsilon}$ of the equation

$$
\begin{cases}u_{\varepsilon}-\frac{1}{\lambda} \operatorname{div} \frac{D u_{\varepsilon}}{\sqrt{\varepsilon^{2}+\left|D u_{\varepsilon}\right|^{2}}}=1 & \text { in } C, \\ u_{\varepsilon}=0 & \text { on } \partial C\end{cases}
$$

such that $0 \leq u_{\varepsilon} \leq 1$. Moreover, there exist $\lambda_{0}$ and $\varepsilon_{0}$, depending only on $\partial C$, such that if $\lambda \geq \lambda_{0}$ and $\varepsilon \leq \varepsilon_{0}$, then $u_{\varepsilon}$ is a concave function such that $u_{\varepsilon} \geq \alpha>0$ on $\partial C$ for some $\alpha>0$. Hence, $u_{\varepsilon}$ satisfies

$$
\left[\frac{D u^{\epsilon}}{\sqrt{\epsilon^{2}+\left|D u^{\epsilon}\right|^{2}}} \cdot v^{C}\right]=\operatorname{sign}\left(0-u^{\epsilon}\right)=-1 \quad \text { on } \partial C \text {. }
$$

As $\varepsilon \rightarrow 0$, the functions $u_{\varepsilon}$ converge to the concave function $u$ minimizing the problem

$$
\min _{u \in B V(C)} \int_{C}|D u|+\int_{\partial C}|u(x)| d \mathscr{H}^{N-1}(x)+\frac{\lambda}{2} \int_{C}|u(x)-1|^{2} d x ;
$$

equivalently, if $u$ is extended with zero out of $C$, the extension minimizes

$$
\int_{\mathbb{R}^{N}}|D u|+\frac{\lambda}{2} \int_{\mathbb{R}^{N}}\left|u-\chi_{C}\right|^{2} d x .
$$

The function $u$ satisfies $0 \leq u<1$. The superlevel set $\{u \geq t\}$, for $t \in(0,1]$, is contained in $C$ and minimizes the problem

$$
\min _{F \subset C} P(F)-\lambda(1-t)|F| \text {. }
$$

It was proved in [Alter et al. 2005a] (see also [Caselles et al. 2005]) that the set $C^{*}=\left\{u=\max _{C} u\right\}$ is the maximal Cheeger set contained in $C$, that is, the maximal set that solves (1). Moreover, one has $u=1-h_{C} / \lambda>0$ in $C^{*}$ and $h_{C}=\lambda_{C^{*}}$.

If we want to consider what happens inside $C^{*}$, and in particular whether there are other Cheeger sets, we have to analyze the level sets of $u_{\varepsilon}$ before passing to the limit as $\epsilon \rightarrow 0^{+}$. To do this, we introduce the following rescaling of $u_{\varepsilon}$ :

$$
v_{\varepsilon}=\frac{u_{\varepsilon}-m_{\varepsilon}}{\varepsilon} \leq 0
$$


where $m_{\varepsilon}=\max _{C} u_{\varepsilon} \rightarrow 1-h_{C} / \lambda$ as $\varepsilon \rightarrow 0$. The function $v_{\varepsilon}$ is a generalized solution of the equation:

$$
\begin{cases}\varepsilon v_{\varepsilon}-\frac{1}{\lambda} \operatorname{div} \frac{D v_{\varepsilon}}{\sqrt{1+\left|D v_{\varepsilon}\right|^{2}}}=1-m_{\varepsilon} & \text { in } C, \\ v_{\varepsilon}=-\frac{m_{\varepsilon}}{\varepsilon} & \text { on } \partial C .\end{cases}
$$

We define the vector field

$$
z_{\varepsilon}=D u_{\varepsilon} / \sqrt{\varepsilon^{2}+\left|D u_{\varepsilon}\right|^{2}}=D v_{\varepsilon} / \sqrt{1+\left|D v_{\varepsilon}\right|^{2}} ;
$$

it lies in $L^{\infty}(C)$, has uniformly bounded divergence, and satisfies $\left|z_{\varepsilon}\right| \leq 1$ a.e. in $C$ and, by (14), $\left[z_{\varepsilon} \cdot v_{C}\right]=-1$ on $\partial C$.

We now study the limit of $v_{\varepsilon}$ and $z_{\varepsilon}$ as $\varepsilon \rightarrow 0$. By the concavity of $v_{\varepsilon}$, for each $\varepsilon>0$ small enough and each $s \in(0,|C|)$, there exists a (convex) superlevel set $C_{s}^{\varepsilon}$ of $v_{\varepsilon}$ such that $\left|C_{s}^{\varepsilon}\right|=s$. Moreover, $\left\{v_{\varepsilon}=0\right\}$ is a null set: otherwise, since $v_{\varepsilon}$ is concave, it would be a convex set of positive measure, hence with nonempty interior. We would then have $v_{\varepsilon}=\operatorname{div} z_{\varepsilon}=0$, hence $1-m_{\varepsilon}=0$ in the interior of $\left\{v_{\varepsilon}=0\right\}$. This is a contradiction with Theorem 4 for $\varepsilon>0$ small enough.

Hence we may take $C_{0}^{\varepsilon}:=\left\{v_{\varepsilon}=0\right\}$ and $C_{|C|}^{\varepsilon}:=C$. The boundaries $\partial C_{s}^{\varepsilon} \cap C$ define a foliation in $C$, in the sense that for all $x \in C$, there exists a unique value of $s \in[0,|C|]$ such that $x \in \partial C_{s}^{\varepsilon}$.

A sequence of uniformly bounded convex sets is compact both for the $L^{1}$ and Hausdorff topologies. Hence, up to a subsequence, we may assume that the $C_{s}^{\varepsilon}$ converge to convex sets $C_{s}$, each of volume $s$, first for any $s \in \mathbb{Q} \cap(0,|C|)$ and then by continuity for any $s$. Possibly extracting a further subsequence, we may assume that there exists $s_{*} \in[0,|C|]$ such that $v_{\varepsilon}$ goes to a concave function $v$ in $C_{s}$ for any $s<s_{*}$, and to $-\infty$ outside $C_{*}:=C_{s_{*}}$. We may also assume that $z_{\varepsilon} \rightarrow z$ weakly* in $L^{\infty}(C)$, for some vector field $z$ satisfying $|z| \leq 1$ a.e. in $C$. From (13) we have in the limit

$$
-\operatorname{div} z=\lambda(1-u) \quad \text { in } \mathscr{D}^{\prime}(C) .
$$

Moreover, $-\operatorname{div} z \in \partial \Psi_{0}(u)$ by the results recalled in Section 2 . We then see from (18) that

$$
-\operatorname{div} z=h_{C} \quad \text { in } C^{*},
$$

while $-\operatorname{div} z>h_{C}$ a.e. on $C \backslash C^{*}$.

Set $s^{*}:=\left|C^{*}\right|$, so $C^{*}=C_{s^{*}}$. By Theorem 4 , for $s \geq s^{*}$, the set $C_{s}$ is a minimizer of the variational problem

$$
\min _{E \subseteq C} P(E)-\mu_{s}|E|
$$


for some $\mu_{s} \geq h_{C}$ ( $\mu_{s}$ is equal to the constant value of $-\operatorname{div} z=\lambda(1-u)$ on $\partial C_{s} \cap C$; see (16)). Notice that $\mu_{s}$ is bounded from above by $P(C) /(|C|-s)$ : indeed,

$$
-\int_{C \backslash C_{s}^{\varepsilon}} \operatorname{div} z_{\varepsilon}(x) d x=\mathscr{H}^{N-1}\left(\partial C \backslash \partial C_{s}^{\varepsilon}\right)-\int_{\partial C_{s}^{\varepsilon} \cap C} \frac{\left|D u_{\varepsilon}\right|}{\sqrt{1+\left|D u_{\varepsilon}\right|^{2}}} \leq P(C)
$$

for $\varepsilon>0$, since the inner normal to $C_{s}^{\varepsilon}$ at $x \in \partial C_{s}^{\varepsilon} \cap C$ is $D u_{\varepsilon}(x) /\left|D u_{\varepsilon}(x)\right|$. On the other hand,

$$
-\int_{C \backslash C_{s}^{\varepsilon}} \operatorname{div} z_{\varepsilon}(x) d x=\int_{C \backslash C_{s}^{\varepsilon}} \lambda\left(1-u_{\varepsilon}(x)\right) d x \geq \mu_{s}^{\varepsilon}(|C|-s),
$$

where $\mu_{s}^{\varepsilon}$ is the constant value of $\lambda\left(1-u_{\varepsilon}\right)$ on the level set $\partial C_{s}^{\varepsilon} \cap C$, and goes to $\mu_{s}$ as $\varepsilon \rightarrow 0$. A more careful analysis would show, in fact, that

$$
\mu_{s} \leq \frac{P(C)-P\left(C_{s}\right)}{|C|-s} .
$$

For $s>s^{*}$, we have $\mu_{s}>h_{C}$ and the set $C_{s}$ is the unique minimizer of the variational problem (20). As a consequence (see [Alter et al. 2005a; Caselles et al. 2005]) for any $s>s^{*}$ the set $C_{s}$ is also the unique minimizer of $P(E)$ among all $E \subseteq C$ of volume $s$.

Lemma 3.1. We have $s_{*}>0$ and the sets $C_{s}$ are Cheeger sets in $C$ for any $s \in$ $\left[s_{*}, s^{*}\right]$.

Proof. Let $s_{*}<s \leq|C|$. If $x \in \partial C_{s}^{\epsilon} \backslash \partial C$, then

$$
0-v_{\varepsilon}(x) \leq D v_{\varepsilon}(x) \cdot\left(\bar{x}_{\varepsilon}-x\right)
$$

where $v_{\varepsilon}\left(\bar{x}_{\varepsilon}\right)=\max _{C} v_{\varepsilon}$. Hence, $\lim _{\varepsilon \rightarrow 0} \inf _{\partial C_{s}^{\varepsilon} \backslash \partial C}\left|D v_{\varepsilon}\right|=+\infty$. Since $\left[z_{\varepsilon} \cdot v^{C}\right]=$ -1 on $\partial C$ and $P\left(C_{s}^{\varepsilon}\right) \rightarrow P\left(C_{s}\right)$, we deduce

$$
\begin{aligned}
-\int_{\partial C_{s}^{\varepsilon}}\left[z_{\varepsilon}(x) \cdot v^{C_{s}^{\varepsilon}}(x)\right] d \mathscr{H}^{N-1}(x) \\
=\int_{\partial C_{s}^{\varepsilon} \backslash \partial C} \frac{\left|D v_{\varepsilon}(x)\right|}{\sqrt{1+\left|D v_{\varepsilon}(x)\right|^{2}}} d \mathscr{H}^{N-1}(x)+\mathscr{H}^{N-1}\left(\partial C_{s}^{\varepsilon} \cap \partial C\right) \rightarrow P\left(C_{s}\right)
\end{aligned}
$$

as $\varepsilon \rightarrow 0^{+}$. Hence,

$$
\begin{aligned}
\int_{\partial C_{s}}\left[z \cdot v^{C_{s}}\right] d \mathscr{H}^{N-1} & =\int_{C_{s}} \operatorname{div} z=\lim _{\varepsilon \rightarrow 0} \int_{C_{s}^{\varepsilon}} \operatorname{div} z_{\varepsilon} \\
& =\lim _{\varepsilon \rightarrow 0} \int_{\partial C_{s}^{\varepsilon}}\left[z_{\varepsilon} \cdot v_{C_{s}^{\varepsilon}}\right] d \mathscr{H}^{N-1}=-P\left(C_{s}\right) .
\end{aligned}
$$


Since $|z| \leq 1$ a.e. in $C$, we deduce that $\left[z \cdot v^{C_{s}}\right]=-1$ on $\partial C_{s}$ for any $s>s_{*}$ (in particular, $|z|=1$ a.e. in $C \backslash C_{*}$ ). Using this and (19), we have for all $s_{*}<s \leq s^{*}$

$$
\frac{P\left(C_{s}\right)}{\left|C_{s}\right|}=h_{C} .
$$

This has two consequences. First, from the isoperimetric inequality, we obtain

$$
h_{C}=\frac{P\left(C_{s}\right)}{\left|C_{s}\right|} \geq \frac{P\left(B_{1}\right)}{\left(\left|B_{1}\right|^{N-1} s\right)^{1 / N}},
$$

if $s \in\left(s_{*}, s^{*}\right]$, so that $s_{*}>0$. Moreover, $C_{s}$ is a Cheeger set for any $s \in\left(s_{*}, s^{*}\right]$, and by continuity $C_{*}$ is also a Cheeger set.

Since the sets $C_{s}$ are convex minimizers of $P(E)-\mu_{s}|E|$ among all $E \subseteq C$, for $s \geq s_{*}$, their boundary is of class $C^{1,1}$ [Brézis and Kinderlehrer 1974; Stredulinsky and Ziemer 1997], with curvature at most $\mu_{s}$, and equal to $\mu_{s}$ in the interior of $C$ (note that $\mu_{s}=h_{C}$ for $s \in\left[s_{*}, s^{*}\right]$ ).

Remark 3.2. Either $s_{*}=s^{*}$, and so $C_{*}=C^{*}$, or $s_{*}<s^{*}$, and so $C^{*}=\bigcup_{s \in\left(s_{*}, s^{*}\right)} C_{s}$. In the latter case, the supremum of the sum $\kappa_{C^{*}}$ of the principal curvatures on $\partial C^{*}$ is equal to $h_{C}$. Indeed, if this were not the case, by considering $C^{\prime} \subset \operatorname{int}\left(C^{*}\right)$ with curvature strictly below $h_{C}$, together with the smallest set $C_{s}$ with $s>s_{*}$ containing $C^{\prime}$, we would get $\kappa_{C^{\prime}}(x) \geq \kappa_{C_{s}}(x)=h_{C}$ at all $x \in \partial C^{\prime} \cap \partial C_{s}$, a contradiction. In particular, $C=C_{*}$ if the supremum of $\kappa_{C}$ on $\partial C$ is strictly less than $P(C) /|C|$; this condition also implies $C=C^{*}$ by [Alter et al. 2005a].

From the strong convergence of $D v_{\varepsilon}$ to $D v$ (in $L^{2}\left(C_{s}\right)$ for any $s<s_{*}$ ), we deduce that $z=D v / \sqrt{1+|D v|^{2}}$ in $C_{*}$. It follows that $v$ satisfies the equation

$$
-\operatorname{div} \frac{D v}{\sqrt{1+|D v|^{2}}}=h_{C} \quad \text { in } C_{*} \text {. }
$$

Integrating both sides of (22) in $C_{*}$, we deduce that

$$
\left[\frac{D v}{\sqrt{1+|D v|^{2}}} \cdot v^{C_{*}}\right]=-1 \quad \text { on } \partial C_{*} .
$$

Lemma 3.3. The set $C_{*}$ is the minimal Cheeger set of $C$; that is, any Cheeger set of $C$ must contain $C_{*}$.

Proof. Let $K \subseteq C^{*}$ be a Cheeger set in $C$. We have

$$
h_{C}|K|=-\int_{K} \operatorname{div} z=-\int_{\partial K}\left[z \cdot v^{K}\right] d \mathscr{H}^{N-1}=P(K),
$$

so $\left[z \cdot v^{K}\right]=-1$ a.e. on $\partial K$. Let $v^{\epsilon}$ and $v$ be the vector fields of unit normals to the sets $C_{s}^{\epsilon}$ and $C_{s}, s \in[0,|C|]$, respectively. By the Hausdorff convergence of $C_{s}^{\epsilon}$ 
to $C_{s}$ as $\epsilon \rightarrow 0^{+}$for any $s \in[0,|C|]$, we have $v^{\epsilon} \rightarrow v$ a.e. in $C$. On the other hand, $\left|z_{\epsilon}+v^{\epsilon}\right| \rightarrow 0$ locally uniformly in $C \backslash \bar{C}_{*}$ : indeed, in $C$,

$$
\left|z_{\epsilon}+v^{\epsilon}\right|=\left|\frac{D v_{\varepsilon}}{\sqrt{1+\left|D v_{\varepsilon}\right|^{2}}}-\frac{D v_{\varepsilon}}{\left|D v_{\varepsilon}\right|}\right|=\left|\frac{\left|D v_{\varepsilon}\right|}{\sqrt{1+\left|D v_{\varepsilon}\right|^{2}}}-1\right| \text {. }
$$

Since $\left|D v_{\epsilon}\right| \rightarrow \infty$ uniformly in any subset of $C$ at positive distance from $C_{*}$ (see the first lines of the proof of Lemma 3.1), this shows the uniform convergence of $\left|z_{\epsilon}+v^{\epsilon}\right|$ to 0 in such subsets.

These two facts imply that $z=-v$ a.e. on $C \backslash C_{*}$. By modifying $z$ in a set of null measure, we may assume that $z=-v$ on $C \backslash C_{*}$. We recall that the sets $C_{s}, s \geq s_{*}$ are minimizers of variational problems of the form $\min _{K \subseteq C} P(K)-\mu|K|$, for some values of $\mu$ (with $\mu=h_{C}$ as long as $s \leq s^{*}$ and $\mu=\mu_{s}>h_{C}$ continuously increasing with $s>s^{*}$ ). Since these sets are convex, with boundary (locally) uniformly of class $C^{1,1}$, and the map $s \rightarrow C_{s}$ is continuous in the Hausdorff topology, we conclude that the normal $v(x)$ is a continuous function in $C \backslash \operatorname{int}\left(C_{*}\right)$.

Since $|z|<1$ inside $C_{*}$ and $\left[z \cdot v^{K}\right]=-1$ a.e. on $\partial K$, by [Anzellotti 1983a, Theorem 1]) we have that the boundary of $K$ must be outside the interior of $C_{*}$, hence either $K \supseteq C_{*}$ or $K \cap C_{*}=\varnothing$ (modulo a null set). Let us prove that the last situation is impossible. Indeed, assume that $K \cap C_{*}=\varnothing$ (modulo a null set). Since $\partial K$ is of class $C^{1}$ out of a closed set of zero $\mathscr{H}^{N-1}$-measure (see [Gonzalez et al. 1983]) and $z$ is continuous in $C \backslash \operatorname{int}\left(C_{*}\right)$, by Theorem 2 we have

$$
z(x) \cdot v^{K}(x)=-1 \quad \mathscr{H}^{N-1} \text {-a.e. on } \partial K .
$$

Now, since $K \cap C_{*}=\varnothing$ (modulo a null set), then there is some $s \geq s_{*}$ and some $x \in \partial C_{s} \cap \partial K$ such that $v^{K}(x)+v(x)=0$. Fix $0<\epsilon<2$. By a slight perturbation, if necessary, we may assume that $x \in \partial C_{s} \cap \partial K$ with $s>s_{*}$, (23) holds at $x$ and

$$
\left|v^{K}(x)+v(x)\right|<\epsilon .
$$

Since by (23) we have $v(x)=-z(x)=v^{K}(x)$ we obtain a contradiction with (24). We deduce that $K \supseteq C_{*}$.

Therefore, in order to prove the uniqueness of the Cheeger set of $C$, it is enough to show that

$$
C_{*}=C^{*} .
$$

Recall that the boundary of both $C_{*}$ and $C^{*}$ is of class $C^{1,1}$, and the sum of its principal curvatures is less than or equal $h_{C}$, and constantly equal to $h_{C}$ in the interior of $C$. We now show that if $C_{*} \neq C^{*}$ and under additional assumptions, the sum of the principal curvatures of the boundary of $C^{*}$ (or of any $C_{s}$ for $s \in\left(s_{*}, s^{*}\right]$ ) must be $h_{C}$ out of $C_{*}$. 
Lemma 3.4. Assume that $C$ has $C^{2}$ boundary. Let $s \in\left(s_{*}, s^{*}\right]$ and $x \in \partial C_{s} \backslash \partial C_{*}$. If the sum of the principal curvatures of $\partial C_{s}$ at $x$ is strictly below $h_{C}$, then the Gaussian curvature of $\partial C$ at $x$ is 0 .

Proof. Let $x \in \partial C_{s} \backslash \partial C_{*}$ and assume the sum of the principal curvatures of $\partial C_{s}$ at $x$ is strictly below $h_{C}$ (assuming $x$ is a Lebesgue point for the curvature on $\partial C_{s}$ ). Necessarily, this implies that $x \in \partial C$. Assume then that the Gauss curvature of $\partial C$ at $x$ is positive: by continuity, in a neighborhood of $x, C$ is uniformly convex and the sum of the principal curvatures is less than $h_{C}$. We may assume that near $x$, $\partial C$ is the graph of a nonnegative, $C^{2}$ and convex function $f: B \rightarrow \mathbb{R}$ where $B$ is an $(N-1)$-dimensional ball centered at $x$. We may as well assume that $\partial C_{s}$ is the graph of $f_{s}: B \rightarrow \mathbb{R}$, which is $C^{1,1}$ [Brézis and Kinderlehrer 1974; Stredulinsky and Ziemer 1997], and also nonnegative and convex. In $B$, we have $f_{s} \geq f \geq 0$, and

$$
D^{2} f \geq \alpha I \text { and } \operatorname{div} \frac{D f}{\sqrt{1+|D f|^{2}}}=h
$$

with $h \in C^{0}(\bar{B}), h<h_{C}, \alpha>0$, while

$$
\operatorname{div} \frac{D f_{s}}{\sqrt{1+\left|D f_{s}\right|^{2}}}=h \chi_{\left\{f=f_{s}\right\}}+h_{C} \chi_{\left\{f_{s}>f\right\}}
$$

(where $\chi_{\left\{f=f_{s}\right\}}$ has positive density at $x$ ).

We let $g=f_{s}-f \geq 0$. Introducing the Lagrangian $\Psi: \mathbb{R}^{N-1} \rightarrow[0,+\infty)$ given by $\Psi(p)=\sqrt{1+|p|^{2}}$, we obtain, for a.e. $y \in B$,

$$
\begin{aligned}
\left(h_{C}-h(y)\right) \chi_{\{g>0\}} & (y) \\
& =\operatorname{div}\left(D \Psi\left(D f_{s}(y)\right)-D \Psi(D f(y))\right) \\
& =\operatorname{div}\left(\left(\int_{0}^{1} D^{2} \Psi\left(D f(y)+t\left(D f_{s}(y)-D f(y)\right)\right) d t\right) D g(y)\right),
\end{aligned}
$$

so that, letting $A(y):=\int_{0}^{1} D^{2} \Psi(D f(y)+t D g(y)) d t$ (which is a positive definite matrix and Lipschitz continuous inside $B$ ), we see that $g$ is the minimizer of the functional

$$
w \mapsto \int_{B}\left(A(y) D w(y) \cdot D w(y)+\left(h_{C}-h(y)\right) w(y)\right) d y
$$

under the constraint $w \geq 0$ and with boundary condition $w=f_{s}-f$ on $\partial B$. Adapting the results in [Caffarelli and Rivière 1976] we get that $\left\{f=f_{s}\right\}=\{g=0\}$ is the closure of a nonempty open set with boundary of zero $\mathscr{H}^{N-1}$-measure.

We therefore have found an open subset $D \subset \partial C \cap \partial C_{s}$, disjoint from $\partial C_{*}$, on which $C$ is uniformly convex, with curvature less than $h_{C}$. Let $\varphi$ be a smooth, nonnegative function with compact support in $D$. One easily shows that if $\varepsilon>0$ is small enough, $\partial C_{s}-\varepsilon \varphi \nu^{C_{s}}$ is the boundary of a set $C_{\epsilon}^{\prime}$ which is still convex, with 
$P\left(C_{\epsilon}^{\prime}\right) /\left|C_{\epsilon}^{\prime}\right|>P\left(C_{s}\right) /\left|C_{s}\right|=h_{C}$ (just differentiate the map $\left.\epsilon \rightarrow P\left(C_{\epsilon}^{\prime}\right) /\left|C_{\epsilon}^{\prime}\right|\right)$, and the sum of its principal curvatures is less than $h_{C}$. This implies that for $\epsilon>0$ small enough, the set $C^{\prime}:=C_{\epsilon}^{\prime}$ is calibrable [Alter et al. 2005a], which in turn implies that $\min _{K \subset C^{\prime}} P(K) /|K|=P\left(C^{\prime}\right) /\left|C^{\prime}\right|$. But this contradicts $C_{*} \subset C^{\prime}$, which is true for $\varepsilon$ small enough.

Proof of Theorem 3. Assume that $C$ is $C^{2}$ and uniformly convex. Let us prove that its Cheeger set is unique. Assume by contradiction that $C^{*} \neq C_{*}$. From Lemma 3.4 we have that the sum of the principal curvatures of $\partial C^{*}$ is $h_{C}$ outside of $C_{*}$.

Let now $\bar{x} \in \partial C^{*} \cap \partial C_{*}$ be such that $\partial C^{*} \cap B_{\rho}(\bar{x}) \neq \partial C_{*} \cap B_{\rho}(\bar{x})$ for all $\rho>$ 0 ( $\partial C^{*} \cap \partial C_{*} \neq \varnothing$ since otherwise both $C^{*}$ and $C_{*}$ would be balls, which is impossible). Letting $T$ be the tangent hyperplane to $\partial C^{*}$ at $\bar{x}$, we can write $\partial C^{*}$ and $\partial C_{*}$ as the graph of two positive convex functions $v^{*}$ and $v_{*}$, respectively, over $T \cap B_{\rho}(\bar{x})$ for $\rho>0$ small enough. Identifying $T \cap B_{\rho}(\bar{x})$ with $B_{\rho} \subset \mathbb{R}^{N-1}$, we have that $v_{*}, v^{*}: B_{\rho} \rightarrow \mathbb{R}$ both solve the equation

$$
-\operatorname{div} \frac{D v}{\sqrt{1+|D v|^{2}}}=f
$$

for some function $f \in L^{\infty}\left(B_{\rho}\right)$. Moreover, it holds $v_{*} \geq v^{*}, v_{*}(0)=v^{*}(0)$ and $v_{*}(y)>v^{*}(y)$ for some $y \in B_{\rho}$. Notice that $f=\lambda_{C}$ in the (open) set where $v_{*}>v^{*}$, in particular both functions are smooth in this set. Let $D$ be an open ball such that $\bar{D} \subset B_{\rho}, v_{*}>v^{*}$ on $D$ and $v_{*}(y)=v^{*}(y)$ for some $y \in \partial D$. Notice that, since both $v^{*}$ and $v_{*}$ belong to $C^{\infty}(D) \cap C^{1}(\bar{D})$, the fact that $v_{*}(y)=v^{*}(y)$ also implies that $D v_{*}(y)=D v^{*}(y)$. In $D$, both functions solve (26) with $f=\lambda_{C}$. Letting $w=v_{*}-v^{*}$, we obtain $w(y)=0$ and $D w(y)=0$, while $w>0$ inside $D$. Recalling the function $\Psi(p)=\sqrt{1+|p|^{2}}$, we have, for any $x \in D$,

$$
\begin{aligned}
0 & =\operatorname{div}\left(D \Psi\left(D v_{*}(x)\right)-D \Psi\left(D v^{*}(x)\right)\right) \\
& =\operatorname{div}\left(\left(\int_{0}^{1} D^{2} \Psi\left(D v^{*}(x)+t\left(D v_{*}(x)-D v^{*}(x)\right)\right) d t\right) D w(x)\right),
\end{aligned}
$$

so that $w$ solves a linear, uniformly elliptic equation with smooth coefficients. Then Hopf's lemma [Gilbarg and Trudinger 1983] implies that $D w(y) \cdot v_{D}(y)<0$, a contradiction. Hence $C_{*}=C^{*}$.

Remark 3.5. As a consequence of Theorem 3 and the results of [Giusti 1978], if $C$ is of class $C^{2}$ and uniformly convex, Equation (22) has a solution on the whole of $C$, if and only if $C$ is a Cheeger set of itself, i.e., if and only if the sum of the principal curvatures of $\partial C$ is less than or equal to $P(C) /|C|$.

Remark 3.6. The results of this paper can be easily extended to the anisotropic setting (see [Caselles et al. 2005]) provided the anisotropy is smooth and uniformly elliptic. 


\section{References}

[Alter and Caselles 2007] F. Alter and V. Caselles, "Uniqueness of the Cheeger set of a convex body", preprint, 2007, Available at benasque.ecm.ub.es/2007pde/talks_contr/072cheeger8.pdf.

[Alter et al. 2005a] F. Alter, V. Caselles, and A. Chambolle, "A characterization of convex calibrable

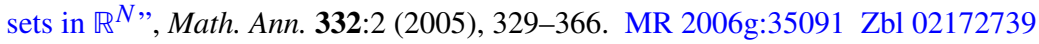

[Alter et al. 2005b] F. Alter, V. Caselles, and A. Chambolle, "Evolution of characteristic functions of convex sets in the plane by the minimizing total variation flow", Interfaces Free Bound. 7:1 (2005), 29-53. MR 2006b:35154 Zbl 1084.49003

[Ambrosio et al. 2000] L. Ambrosio, N. Fusco, and D. Pallara, Functions of bounded variation and free discontinuity problems, Oxford University Press, New York, 2000. MR 2003a:49002 Zbl 0957.49001

[Andreu et al. 2001] F. Andreu, C. Ballester, V. Caselles, and J. Mazón, “The Dirichlet problem for the total variation flow", J. Funct. Anal. 180:2 (2001), 347-403. MR 2001k:35143 Zbl 0973.35109

[Anzellotti 1983a] G. Anzellotti, "Pairings between measures and bounded functions and compensated compactness", Ann. Mat. Pura Appl. (4) 135 (1983), 293-318 (1984). MR 85m:46042 Zbl 0572.46023

[Anzellotti 1983b] G. Anzellotti, "Traces of bounded vector fields and the divergence theorem", preprint, Dipartimento di Matematica, Università di Trento, 1983.

[Bellettini et al. 2002] G. Bellettini, V. Caselles, and M. Novaga, "The total variation flow in $\mathbb{R}^{N}$ ", J. Differential Equations 184:2 (2002), 475-525. MR 2003g:35105 Zbl 1036.35099

[Brézis and Kinderlehrer 1974] H. Brézis and D. Kinderlehrer, "The smoothness of solutions to nonlinear variational inequalities", Indiana Univ. Math. J. 23 (1974), 831-844. MR 50 \#13881 Zbl 0278.49011

[Caffarelli and Rivière 1976] L. A. Caffarelli and N. M. Rivière, "On the rectifiability of domains with finite perimeter", Ann. Scuola Norm. Sup. Pisa Cl. Sci. (4) 3:2 (1976), 177-186. MR 53 \#14288 Zbl 0362.49031

[Caselles et al. 2005] V. Caselles, A. Chambolle, S. Moll, and M. Novaga, "A characterization of convex calibrable sets in $\mathbb{R}^{N}$ with respect to anisotropic norms", preprint, 2005.

[Gilbarg and Trudinger 1983] D. Gilbarg and N. S. Trudinger, Elliptic partial differential equations of second order, 2nd ed., Grundlehren der Math. Wissenschaften 224, 1983. Corrected reprint, 1998. MR 2001k:35004 Zbl 0562.35001

[Giusti 1976] E. Giusti, "Boundary value problems for non-parametric surfaces of prescribed mean curvature”, Ann. Scuola Norm. Sup. Pisa Cl. Sci. (4) 3:3 (1976), 501-548. MR 58 \#2572 Zbl 0344. 35036

[Giusti 1978] E. Giusti, "On the equation of surfaces of prescribed mean curvature. Existence and uniqueness without boundary conditions", Invent. Math. 46:2 (1978), 111-137. MR 58 \#7337 Zbl 0381.35035

[Gonzalez et al. 1983] E. Gonzalez, U. Massari, and I. Tamanini, "On the regularity of boundaries of sets minimizing perimeter with a volume constraint", Indiana Univ. Math. J. 32:1 (1983), 25-37. MR 84d:49043 Zbl 0486.49024

[Kawohl and Kutev 1995] B. Kawohl and N. Kutev, "Global behaviour of solutions to a parabolic mean curvature equation”, Differential Integral Equations 8:8 (1995), 1923-1946. MR 96f:35088 Zbl 0844.35050

[Kawohl and Lachand-Robert 2006] B. Kawohl and T. Lachand-Robert, "Characterization of Cheeger sets for convex subsets of the plane”, Pacific J. Math. 225:1 (2006), 103-118. MR 2007e:52002 Zbl 05170768 
[Kawohl and Novaga 2006] B. Kawohl and M. Novaga, "The $p$-Laplace eigenvalue problem as $p \rightarrow 1$ and Cheeger sets in a Finsler metric", preprint, 2006, Available at http://cvgmt.sns.it/papers/ kawnov06/KawNovFeb27.pdf.

[Lichnewsky and Temam 1978] A. Lichnewsky and R. Temam, "Pseudosolutions of the time-dependent minimal surface problem”, J. Differential Equations 30:3 (1978), 340-364. MR 80a:35059 Zbl 0368.49016

[Stredulinsky and Ziemer 1997] E. Stredulinsky and W. P. Ziemer, "Area minimizing sets subject to a volume constraint in a convex set”, J. Geom. Anal. 7:4 (1997), 653-677. MR 99k:49089 Zbl 0940.49025

Received April 6, 2006. Revised July 21, 2006.

\author{
Vicent CASElles \\ DEPARTAMENT DE TECNOLOGIA \\ UNIVERSITAT POMPEU FABRA \\ PASSEIG CiRCUMVALlació, 8 \\ 08003 BARCELONA \\ SPAIN \\ vicent.caselles@upf.edu \\ ANTONIN CHAMBOLLE \\ CMAP, ÉCOLE PolytechniQue \\ CNRS \\ 91128 PALAisEaU \\ FRANCE \\ antonin.chambolle@polytechnique.fr \\ Matteo Novaga \\ DipARTIMENTO Di MATEMATICA \\ UNIVERSITÀ DI PISA \\ LARGo Bruno PonteCORVO 5 \\ 56127 PISA \\ ITALY \\ novaga@dm.unipi.it
}

